\title{
Teletext system
}

For Teletext, the codewords are transmitted in serial form, one bit at a time.

The codewords are transmitted during the field blanking period during lines 17 and 18 for odd fields and lines 330 and 331 for even fields (Internationally agreed lines).

However, to increase the amount of transmitted data per channel, additional lines may be used during the blanking period, i.e. lines $7-20$ and lines $320-335$

Reference figure 4.6, the video signal, which includes the codewords, is fed from the television video demodulator stage to the Teletext unit. It is the function of this unit to:

Remove the codeword information from the video signal.

Recognise when the transmitted page of Teletext is the

same as the page number the user has entered into the unit via his keyboard.

Control the write address generator so that the received codewords are written into the RAM in the correct location.

The Teletext unit of figure 4.6 therefore takes the place of the keyboard of figure 4.1 (page 100).

Before looking in some detail at the circuitry of the Teletext unit, it is necessary to provide some details of the Teletext transmitted signal.

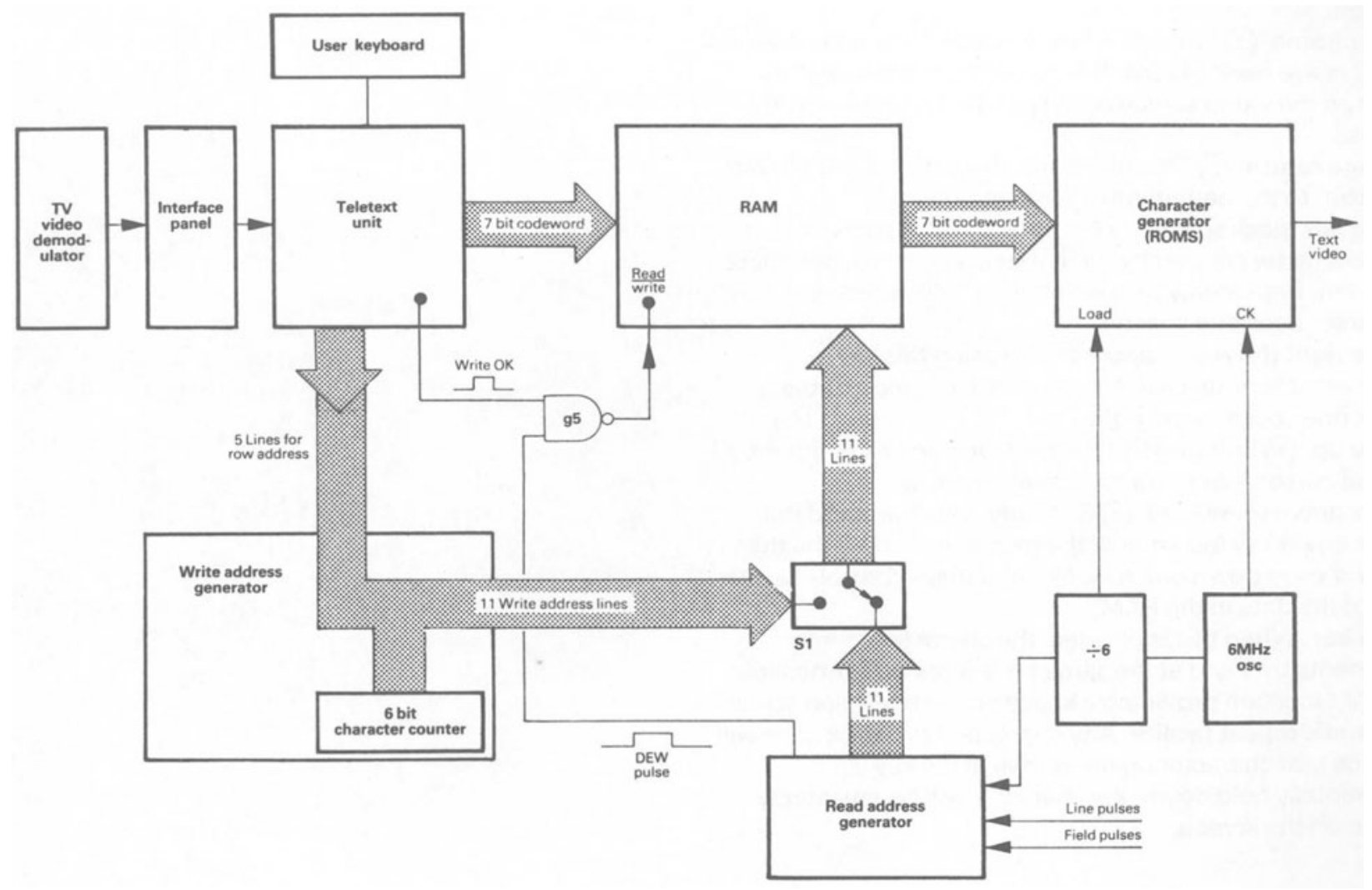

